\title{
Types of Earthenwares and its Uses
}

\author{
M. Anbarasu \\ Post Doctoral Fellow, Department of Agronomy (Farm) \\ Agricultural College and Research Institute, Madurai, Tamil Nadu, India \\ (D) https://orcid.org/0000-0001-7970-4454
}

\section{OPEN ACCESS}

Manuscript ID:

ASH-2020-08023323

Volume: 8

Issue: 2

Month: Ocotober

Year: 2020

P-ISSN: 2321-788X

E-ISSN: 2582-0397

Received: 03.08.2020

Accepted: 15.09.2020

Published: 01.10.2020

\section{Citation:}

Anbarasu, M., and NK

Sathyamoorthy. "Types of Earthenwares and Its Uses."

Shanlax International

Journal of Arts, Science and Humanities, vol. 8 , no. 2,2020 , pp. 107-112.

DOI:

https://doi.org/10.34293/ sijash.v8i2.3323

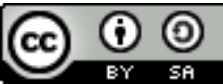

This work is licensed under a Creative Commons Attribution-ShareAlike 4.0 International License

\section{N.K. Sathyamoorthy}

Assistant Professor, Agricultural College and Research Institute, Coimbatore, Tamil Nadu, India

\section{Abstract}

Earthenware is proof of our way of life improvement in an archeological manner. Ceramics is intricate of water, soil, and mud compound utilized as home vessels of old periods. Ceramics making has become a preservationist industry in numerous pieces of the world. This industry is called ceramics in Tamil, and stoneware is classified "Potter." Pot has fundamentally in mud porcelain or (Kaolinite), mud normally contains $40 \%$ aluminum oxide, $46 \%$ silicon oxide, and $14 \%$ water may be all things considered insinuated as having utilization is a lot of medical advantages. Earthenware is organized using a variety of strategies like carefully assembled and potter's wheel strategy. In old Tamilians was fabricated numerous sorts of pots are utilized different purposes and satisfied their needs.

Keywords: Earthenware, Pot, Potter, Clay and Types of Pots

\section{Introduction}

Earthenware is the sort of material that people can use to consider their requirements by utilizing earth. Pottery usually refers to materials made of soil. Pottery is making techniques that have been discovered since ancient times. Pottery making has become a very conservative industry in many parts of the world. This industry is called pottery in Tamil, and pottery is called "Potter."

Water, soil, clay be added to the soil in shape, doing it in a kiln to lead the high-temperature heater are made of pottery. By heating, this results in permanent changes such as tightening of the clay, strength additions, and shape stability. The pottery used for pottery differs from place to place, and the pottery made there is unique. There are some other minerals to be added to the clay soil for certain purposes.

\section{History}

A huge piece of earthenware history depends on ancient, pre-abstract archaeological culture. Quite a bit of this history is in this manner got from relics accessible in archaic exploration. Since stoneware is truly strong, earthenware production and tile shells have been made due for a great many years and are accessible at archeological locales.

Before turning part of the pottery or pottery industry culture, numerous levels must be met. They are

First, pottery should be available. Archaeological sites where the earliest ceramics were found are located close to places where clay sources are readily available. Since China has many types of clay, it can be a pioneer in pottery or art. Not only China, but many other countries have large forms of different clay types. 
Secondly, it must have been possible to create a transitional temperature from crude clay to charcoal. Methods for reliably producing temperatures suitable for burning ceramics are not known until after the development of culture.

Third, there should be enough time to prepare, mold, and bake clay vases. Even after humans knew how to control fire, humans did not know how to create pottery until they could live in a stable place. Pottery is likely to be created only after humans specialize in farming and lead to permanent settlement. However, the earliest known pottery is probably from China before 20,000 BC, before all agriculture was known.

Fourth, it can only be justified about the resources for making ceramics if it was necessary for its production.

\section{Raw Material}

Earthenware has basically in mud porcelain or (Kaolinite) is; Clay ordinarily contains $40 \%$ aluminum oxide, $46 \%$ silicon oxide, and $14 \%$ water might be by and large alluded to as having. Two kinds of mud are found in nature. They are called essential and auxiliary muds. The essential dirt is found on the site of the stone from which it was acquired. It isn't conveyed by running water or ice sheets; it isn't blended in with some other sedimentary structures. The essential earth is substantial, thick, and clean. Auxiliary, or sedimentary dirt, is framed in a lighter silt structure lowered and put away. This second sort of dirt, sedimentary synthesis, is better and lighter than the essential mud. Various added substances give various properties to earth.

- Bronze or Kaolinite: Since it was first used in China, it is sometimes called Chinese clay. It is used in ceramic making.

- Ball Clay: Very fine-grained, fine particulate, sedimentary clay, which may contain some organic matter. During the manufacture of porcelain, small amounts are added to increase its flexibility.

- Theekkaliman: slightly less than the percentage of these clay but is consistent with regular and adequate flexible nature. This type of clay is heat tolerant and increases the heat tolerance of the clay in combination with other types of clay.
- Maakkal Paandak Clay: This type of clay is suitable for making mackerel varieties. The properties of the clay are intermediate between the properties of the clay and the properties of the ball clay. These clays are microscopic particles, like ball clays, and are heat-like.

- Common red clay and silt clay contain vegetable and ferric oxide contaminants useful for making bricks. Still, only a few steps are suitable for making ceramics under special conditions. Besides, in general, such clay ceramics are not suitable for manufacture.

- Bentonite type of clay is highly flexible. This type of clay is added to increase the flexibility of other types of clays.

\section{The Steps of Production}

Clay ponds acquire different physical properties when making ceramics.

- Green vases refer to baked goods. With sufficient moisture, the materials are very flexible. (That is, they are soft and flexible and can be transformed)

- Skin-Weakness refers to the state of the partially dried material made of clay. In this case, the clay material is found to be approximately $15 \%$ moisture content. Clay products found in such a condition are very stable and only very small. Upgrading and handle additions are performed in a skin-tight manner.

- Extremely dry (humid) conditions indicate a relative humidity level of $0 \%$ or more. The ceramics of this stage are incinerated in the furnace to achieve a pinkish brown.

- Baked clay refers to the state in which the substance is burned for the first time after being brought to its desired shape. Baked loaf or bread refers to the baked state. The kiln thus changes the clay material in many ways. Mineral constituents of clay-based material change the color of the material according to the chemical change.

- Glazed or marble baked clay is the final stage in pottery making. A kind of toothpaste is coated with baked clay pottery. Moreover, pottery can be decorated in a variety of ways. The level of pottery thus obtained is a condition of glazed baked clay. When the marble is fired, the fuse adheres well to the pantomime. This kind of 
polishing shooter makes the Phantom even harder.

\section{Methods of Designing}

Potteries are designed using a variety of methods. Some of them are:

1. Handmade Design: This is an early method. Clay Pandas are made from clay coils. Clay bonds are formed by combining flat layers of clay or by bonding and bonding clay solid balls. Thus the parts of the hand-made utensils are often joined together with clay and water-hung hose with the help of the ace. The clay pantry can be decorated with a kiln before or after it is baked.

Figure 1: Handmade and potter's wheel

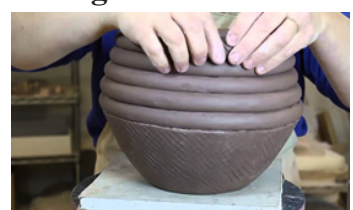

Handmade Design

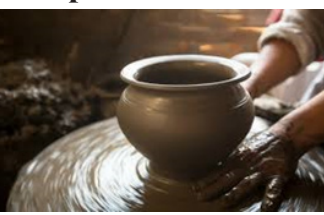

Potter's Wheel

2. Potter's Wheel: In a process known as "throwing," the clay is placed in the middle of a spinning wheel called the wheelbarrow. The pot maker rotates the wheel with a stick, using the keys in his legs or using an electric motor. During this process, the wheel is rotated, the clay core of thin clay is pressed, squeezed, and gently pulled upward and outward into a vacuum. The first step is to press the clay ball downward and inward using a symmetric rotation of a random clay ball. This is a very important step that requires a lot of skill. Forming (creating a hollow sphere with a solid clay ball in the center), Significant skill, and experience to handle the process come to throwing on the wheel are required. Making pottery with acceptable standards requires significant skill and experience to handle the throwing process at the potter's wheel. Pandas were created with such experience and ability to create high-quality elegance. Making pottery with acceptable standards requires significant skill and experience to handle the throwing process at the potter's wheel. Pandas were created with such experience and ability to create high-quality elegance.

\section{Pot Types}

Some of the Types of Pot Offered in Tamil Nadu State.

\begin{tabular}{|c|c|c|c|}
\hline S.No & Tamil Name & English Name & Explanation \\
\hline 1 & அ. .கப்பானை & Ceramic pot & A pot that appears in the ceramic nature \\
\hline 2 & அ. .குப்பானை & Ultra-pot & A pot that appears in the mouth and at the bottom \\
\hline 3 & அகட்டுப்பானை & Pitcher pot & A pitcher like in the middle of pot \\
\hline 4 & அடிசிற்பானை & Adhesive pot & A pot used for brewing \\
\hline 5 & அடுக்குப்பானை & Stacked pot & An array of stacked pots overlapping in moderation or inversion \\
\hline 6 & அரசாணிப்பானை & Rajanippanai & $\begin{array}{l}\text { A pot of gold that is placed on the royal leg of the wedding } \\
\text { ceremony }\end{array}$ \\
\hline 7 & உசும்பியபானை & Usambian pot & A pot was tall \\
\hline 8 & உறிப்பானை & Suction pot & A pot absorbing nature \\
\hline 9 & எ.:குப்பானை & Steel Pot & A pot having mixed Iron \\
\hline 10 & எழுத்துப்பானை & Character pot & A pot drawn on letters \\
\hline 11 & எழுப்புப்பானை & Raising pot & A tall pot \\
\hline 12 & ஒறுவாயப்பானை & Faucet pot & A fringe decay pot \\
\hline 13 & ஓதப்பானை & Wet pot & A pot having wet by surrounded \\
\hline 14 & ஓர்மப்பானை & Orma pot & A pot that sounds good with a flattened \\
\hline 15 & ஓரிப்பானை & Orif pot & A separate pot, a lean pot \\
\hline 16 & ஓவியப்பானை & Portrait pot & A painted pot, painted pot \\
\hline 17 & கஞ்சிப்பானை & Porridge pot & A wide pot used to brew porridge \\
\hline
\end{tabular}




\section{SHanlax}

International Journal of Arts, Science and Humanities

\begin{tabular}{|c|c|c|c|}
\hline 18 & கட்டப்பானை & Ground pot & A bottomless wooden pot \\
\hline 19 & கட்டுப்பானை & Boundary pot & A pot of ammunition for setting a float \\
\hline 20 & கதிர்ப்பானை & Radiator pot & A pot used to hold rice paddies \\
\hline 21 & கரகப்பானை & Cockpit pot & A pot used to ancient folk dance \\
\hline 22 & கரிப்பானை & Charcoal pot & A charcoal favourite pot \\
\hline 23 & கருப்புப்பானை & Black pot & A completely purple pot \\
\hline 24 & கருப்பு-சிவப்பு பானை & Black-red pot & A darker pot on the inside \\
\hline 25 & கலசப்பானை & Jar pot & A pot used to folk god functions \\
\hline 26 & கழுநீர்ப்பானை & Jar & A pot having a lid and filled water \\
\hline 27 & காடிப்பானை & Quail pot & A pot have small brown colour \\
\hline 28 & காதுப்பானை & Ear pot & A pot that can be grasped at the edge \\
\hline 29 & குண்டுப்பானை & Bomb pot & A pot that appears in a rolled-out form \\
\hline 30 & குறைப்பானை & Low Pot & A pot has Bottomless \\
\hline 31 & கூடைப்பானை & Basket Pot & A pot created in the form of a basket \\
\hline 32 & கூர்முனைபானை & Spikes pot & A pot created to form spikes at the base \\
\hline 33 & கூர்ப்பானை & Sharp pot & A pot has sharp end \\
\hline 34 & கூழ்ப்பானை & Pulp & A pot used to brew pulp \\
\hline 35 & கோளப்பானை & Spherical pot & A rolling pot \\
\hline 36 & சருவப்பானை & Slurping pot & $\begin{array}{l}\text { A surface made of pudding - a pitcher made to shrink and shrink } \\
\text { the bottom }\end{array}$ \\
\hline 37 & சவப்பானை & Sawpani & A large pot made from a funeral python \\
\hline 38 & சவலைப்பானை & Crock pot & A well-cooked pot and thin \\
\hline 39 & சன்னப்பானை & Sunny pot & A thin pot and heavy \\
\hline 40 & சாம்பல்பானை & Gray pot & A hand made ash colour pot \\
\hline 41 & சொண்டுப்பானை & Drip pot & A heavy duty pot \\
\hline 42 & சோற்றுப்பானை & Meals pot & A pot used for meals storage \\
\hline 43 & சில்லுப்பானை & Chip pot & A very smallest pot \\
\hline 44 & சின்னபானை & Small pot & A smallest pot \\
\hline 45 & தவலைப்பானை & Frog Pot & A small Pot (Helps to Save Water) \\
\hline 46 & திடமப்பானை & Solid Pot & A large Pot (Solid Pot) \\
\hline 47 & திம்மப்பானை & Thimble Pot & At Most (Thyme - Size) \\
\hline 48 & துந்திப்பானை & Tundra pot & A pot that looks like a tortilla \\
\hline 49 & தொண்ணைப்பானை & Nosy pot & A cavity pot \\
\hline 50 & தோரணப்பானை & Posture pot & A pot used to posture \\
\hline 51 & தோள்பானை & Shoulder Pot & A pot that can be hung on the shoulder \\
\hline 52 & நாற்கால்பானை & Quadruple pot & A pot set with four toes \\
\hline 53 & பச்சைப்பானை & Green pot & A pot used to storage cooked products \\
\hline 54 & படரப்பானை & Creep pot & A pot strong creep - large pot \\
\hline 55 & பிணப்பானை & Carcass pot & A pot used to carcass related one \\
\hline 56 & பொள்ளற்பானை & Pollen pot & A drilled pot (hollow pot) \\
\hline
\end{tabular}




\begin{tabular}{|l|l|l|l|}
\hline 57 & மொங்கல்பானை & Pongal pot & The Pongal Festival pot \\
\hline 58 & மங்கலக்கூலப்பானை & $\begin{array}{l}\text { Mangalak } \\
\text { kolap pot }\end{array}$ & A pot of wine to be used in wedding ceremonies \\
\hline 59 & மடைக்கலப்பானை & Pottery pot & A pot created for cooking in the home or in monasteries or temples \\
\hline 60 & மிண்டப்பானை & Mint Pot & A large size pot \\
\hline 61 & மிறைப்பானை & Bamboo pot & A high-rise pot \\
\hline 62 & முகந்தெழுபானை & Faceplate & A mount like pot \\
\hline 63 & முடனைப்பானை & Mud pot & A round shape pot \\
\hline 64 & முரகுப்பானை & Crude pot & A large pot (rolled pot) \\
\hline 65 & மொங்கம்பானை & Monk Pot & The Great Pot (Mongan Pot) \\
\hline 66 & மொட்டைப்பானை & A terrace pot & A neck less pot \\
\hline 67 & வடிநீர்ப்பானை & Drainage pot & A waterproof water tank \\
\hline 68 & வைைப்பானை & Flavoured Pot & A fresh pot \\
\hline 69 & வெள்ளாவிப்பானை & White pot & A pot that can be used to dry cloth \\
\hline
\end{tabular}

\section{Benefits of Earthenware Usages}

- Earth pot's permeable nature permits both dampness and warmth to course through the food, which brings about moderate yet fragrant food. It additionally holds the sustenance of the food, which is commonly lost in different sorts of utensils. The warm latency in mud pots assists meats with remaining delicate and delicate as the muscle proteins denature and collagen separates.

- Due to its heat resistance and slow cooking, the food retains all its oils and moisture; therefore, you wouldn't require extra oil and fat for providing moisture to your food.

- The moderate cooking and permeable nature of mud pots, the dampness, and fragrance will, in general, remain in the pot without losing any supplement, consequently making it flavorsome. It likewise has a hearty flavor added to it, which we wager you may not get in some other utensil.

- The health benefits of cooking in a clay pot are vast. Firstly, clay pots add many important nutrients like calcium, phosphorous, iron, magnesium, and sulfur to food, which is extremely beneficial to our body. Clay is also alkaline, and thus, acts neutralized the acidity in the food, which makes it easier for us to digest. Importantly, oil is not necessary for cooking in a clay pot, and thus, it is observed that food cooked in clay pots are much lower in fat than food prepared in any other method.
- Since Clay is soluble and prepared, it kills the PH parity of food and thus goes about as a characteristic detox. You will be amazed to realize that mud contains all the potential nutrients, even Vitamin B12.

- Warming the food consistently prompts loss of nourishment, yet if you cook in an earth pot, it holds the temperature for a more drawn out time and no concerns for warming.

- Chief medical advantages of dirt pot cooking originate from its capacity to course steam all through cooking. This gives a lot of dampness and implies that you can cook with less oil and fat.

\section{Conclusion}

Earthenware assumes a significant job in contemplating society and recreating the past. Verifiably with a particular culture, the style of earthenware changed. It mirrors the social, monetary, and natural conditions a culture flourished in which helps the archeologists and students of history in the comprehension of our past. The Tamilians are mentioning the earthenware important and many types manufactured to fulfill their home vessel's needs. Also, while using pots are good health benefits of humans. This article mainly to transfer the knowledge about usage benefits of earthenware types and uses to the upcoming generation. 


\section{References}

Barnett, William K., and John W. Hoopes. The Emergence of Pottery: Technology and Innovation in Ancient Societies, Smithsonian Institution Press, 1995.

"Health Benefits of Clay Pot Cooking." Vegan First, https://www.veganfirst.com/article/healthbenefits-of-clay-pot-cooking

https://www.cfi.de

https://www.facebook.com/worldwidedevangar/ posts/490607171329086

Odelli, E., et al. "Pottery Production and trades in Tamil Nadu Region: New Insights from Alagankulam and Keeladi Excavation Sites." Heritage Science, vol. 8, 2020.
Ryan, William, and C. Radford. Whitewares: Production, Testing and Quality Control: Including Materials, Body Formulations, and Manufacturing Processes, Pergamon Press, 1987.

Vasuki. “பானை - மண்பானை என்று சொல்கிறாமே, அதில் தமிழா்கள் எத்தனை வகை வைத்து இருந்தனா என்று தெரியுமா?" உலகத் தமிழா பேரவை, 2017, https://worldtamilforum.com/ historical_facts/clay-pot/

“மண் பானை.” Wikipedia, https://ta.wikipedia.org/ wiki/மண்_பானை

“மண்பாண்டங்கள் பற்றிய வரலாற்று." Arogyame Selvam, 2018, https://www.arogyameselvam. in/mud-clay-pots/

“பானை வகைகள்.” Wikipedia, https://ta.wikipedia. org/wiki/பானை_வகைகள்

\section{Author Details}

M. Anbarasu, Post Doctoral Fellow, Department of Agronomy (Farm), Agricultural College and Research Institute, Madurai, Tamil Nadu, India, Email ID: manbarasu102@gmail.com.

N.K. Sathyamoorthy, Assistant Professor, Agricultural College and Research Institute, Coimbatore, Tamil Nadu, India,Email ID: nksacrc@gmail.com. 